\title{
Anti-tumor Effects Elicited by Heat Shock Protein 72 Functional Peptide and Alpha-fetoprotein Epitope Peptide Vaccine
}

\author{
Xiaoping Wang ${ }^{\mathrm{a}^{*}}$, Qiaoxia Wang ${ }^{\mathrm{b}}$, Bing $\mathrm{Xu}^{\mathrm{c}}$ and $\mathrm{Na}$ Chang ${ }^{\mathrm{d}}$ \\ Key lab of Molecular Pathology, Shaanxi University of Chinese Medicine, Xianyang 712046, China \\ awxpphd@aliyun.com, b120145557@qq.com, cxubing-95@163.com, d825865603@qq.com \\ ${ }^{*}$ The corresponding Author
}

Keywords: Heat shock protien 72 (HSP72); Alpha-fetoprotein (AFP); Peptide; Tumor; Vaccine

\begin{abstract}
Objective: Alpha-fetoprotein (AFP) is an oncofetal antigen during hepatocellular carcinoma (HCC) development which could lead to weak reproducible antitumor immunity, and may act as a target for cancer therapy. Therefore, it is imperative to enhance its immunogenicity and develop therapeutic vaccines to eliminate AFP-expressing tumors. The study is intended to develop a specific anti-tumor peptide vaccine-heat shock protien 72 functional peptide (HSP72-P) and alpha-fetoprotein epitope peptide (AFP-P). Methods: By way of glutaraldehyde cross-linking, we constructed a potential therapeutic peptide vaccine, HSP72-P/AFP-P. In vitro and in vivo cytotoxicity assays were uesd to verify anti-tumor effect of the recombined vaccine. Results: The results showed that cross-linking of AFP epitope peptide with HSP72 functional peptide induced significant enhancement in specific AFP $\mathrm{CD}^{+} \mathrm{T}$ cells response and distinct cytotoxic antitumor reaction against AFP-expressing tumors. Primimg mice with the reconstructed vaccine, we elicited robust strong protective immunity. Conclusion: Our study suggests that tumor vaccination through cross-linking tumor antigen peptide and HSP72 functional peptide is an encouraging method for cancer immunotherapy.
\end{abstract}

\section{Introduction}

Hepatocellular carcinoma (HCC) is increasing rapidly in Asia because the hepatitis B and C infections spread [1,2]. Currently, the treatments of HCC, usually fail to stop the disease progression due to the growth of residual cancer cells which have escaped from the effective therapies. Although liver transplantation is the effective treatment, most patients still lost chances for the late diagnosis or liver dysfunction under the condition of cirrhosis [1]. The common reasons for mortality in HCC patients contain metastasis, recurrence and the progression of residual primary cancer cells [2]. Novel therapies for HCC should be applied. A combined treatment is probably prolonging patients' life and living quality. In the recent years, immunotherapy with therapeutic immunization vaccine which could supervise and increase the host's immune effect to elicit antigenspecific reaction to HCC, has widely attracted concerns .

Most concerns have focused on cancer immunity which aims to elicit hosts' immune protection against tumor cells. $80 \%$ of primary liver cancers express a high rate of alpha-fetoprotein (AFP), which may act as a target for cancer therapy [3-5]. AFP is an oncofetal antigen during HCC progression which could induce weak reproducible antitumor effect. Therefore, a vaccine specifically targeting AFP is desirable. AFP oncofetal antigen is essential to the promotion and maintenance of cancer cell transformation, which could be an ideal target antigen for AFP vaccine [6,7]. However, AFP immunization has been restricted by improper antigen-specific immune reaction. Therefore, therapies to generate more potential immunotherapy need to be completely explored.

Heat shock proteins (HSP) have been verified to serve as potential immunoadjuvant to increase antigen-specific tumor reaction [8,9]. Thus, an innovative method combining with HSP families will probably produce more potential antitumor immunity $[10,11]$. In the present study, we studied whether AFP epitope peptide (AFP-P) immunogenicity could be enhanced by HSP72 functional peptide (HSP72-P) and whether HSP72-P could increase the potency of AFP-P immunity and 
assessed the immune reaction elicited by HSP72-P/AFP-P. We constructed a tumor vaccine including HSP72 functional peptide and AFP peptide by way of glutaraldehyde cross-linking. Our results showed that AFP peptide and HSP72 functional peptide exhibited distinct enhancement in AFP-P specific $\mathrm{CD}^{+} \mathrm{T}$ cells response and encouraging antitumor immunity against AFP expressing tumors. Primimg mice with the reconstructed vaccine, we elicited robust strong protective immunity. Therefore, cross-linking HSP72-P/AFP-P peptide provides a promise immunotherapy for hepatocellular carcinoma.

\section{Materials and Methods}

AFP peptide, HSP72 Functional Peptide and Conjugation. Mouse AFP peptide was provided by the GenWay Inc. (San Diego, CA, USA). Lyophilized reagents were resuspended, aliquoted in disinfected distilled water and stored at $-70{ }^{\circ} \mathrm{C}$ until use.

Escherichia coli vector expressing mouse HSP72 functional peptide was a gift from Dr. Q Wang in Xi'an Jiaotong University, China. The purified HSP72 protein was identified by Western blotting with a polyclonal antibody (Santa Cruz Inc., CA, USA). The concentration of protein was detected using BCATM protein assay kit (Pierce Inc., IL, USA).

One milligram HSP72 functional peptide was cross-linked to $1 \mathrm{mg}$ AFP peptide in the setting of $0.2 \%$ glutaraldehyde in PBS for $2 \mathrm{~h}$, and dialyzed in PBS for $12 \mathrm{~h}$. The cross-linking complexes aliquots were stored at $-70{ }^{\circ} \mathrm{C}$.

Mice and Cell Line. Female BALB/C mice (6-8 weeks old) were provided by the Experimental Animal Center at Xian Jiaotong University. All mice were kept at pathogen free conditions, and all processes were carried on in accordance with protocols for the correct treatment of research animals. The research was guaranteed by the Ethics Committee of animal Study at Shaanxi University of Chinese Medicine (2004-4B). Mice AFP-producing H22 hepatocarcinoma cell line was a gift from College of Biomedicine, Xi' an Jiaotong University and maintained at $37{ }^{\circ} \mathrm{C}$ under humidified atmosphere of $95 \%$ air and 5\% CO2 in RPMI 1640 with $10 \%$ fetal bovine serum, $100 \mathrm{U} / \mathrm{ml}$ penicillin, and $100 \mu \mathrm{g} / \mathrm{ml}$ streptomycin.

Mice Vaccinated with HSP72-P/AFP-P Complexes. All the BALB/C mice were classified into HSP72-P/AFP-P, AFP-P, HSP72-P and PBS groups. Each group contained ten mice. Before injection, every group was diluted in $0.9 \%$ natural saline to $100 \mu \mathrm{g} / 100 \mu \mathrm{l}$. All the groups were injected subcutaneously (s.c.) to the left flank. Priming and boosting was carried out with $10 \mu \mathrm{g}$ HSP72-P/AFP-P, AFP-P or HSP72-P, while PBS was used as a control. Mice were immunized s.c. with above mentioned vaccines twice every two weeks after the first immunization. Fourteen days after last vaccination, splenic cells were obtained and diluted to different concentrations.

ELISPOT and ELISA Detection. The ELISPOT detection was applied to examine the quantity of specific $\mathrm{T}$ cells generating IFN- $\gamma$ in splenocytes obtained from above vaccinated mice. BD ELISPOT Plates (BD PharMingen, San Diego, CA, USA) were covered with $5 \mu \mathrm{g} / \mathrm{ml}$ rat antimouse IFN- $\gamma$ antibody. After incubated at $4{ }^{\circ} \mathrm{C}$ overnight, the plate was washed in PBS and blocked with RPMI 1640 medium. $1 \times 10^{6}$ splenic cells were put to the ELISPOT plate together with 5 $\mu \mathrm{g} / \mathrm{ml}$ AFP peptide, $10 \mathrm{U} / \mathrm{ml}$ of human interleukin (IL)-2. After cultured at $37^{\circ} \mathrm{C}$ for $24 \mathrm{~h}$, the wells were washed and then incubated with $2.5 \mu \mathrm{g} / \mathrm{ml}$ biotinylated IFN- $\gamma$ antibody overnight at $4^{\circ} \mathrm{C}$. After washing, the plate was incubated with avidin labeled HRP at room temperature for $1 \mathrm{~h}$. After washing for $30 \mathrm{~min}$, spots were generated by staining $100 \mu \mathrm{l}$ 5-bromo-4-chloro-3-indolyl phosphatase / Nitro Blue Tetrazolium (Boehringer Mannheim, IN, USA). The spots in color indicating IFN- $\gamma$ producing cells, were examined under an ELISPOT Reader System.

To determine the anti-AFP antibody level of mice, the serum of mice were detected by ELISA. Ninety-six wells of a microplate were covered with $100 \mu \mathrm{l}$ of $5 \mu \mathrm{g} / \mathrm{ml}$ AFP peptide and cultured overnight at $4{ }^{\circ} \mathrm{C}$. Then the wells were blocked with 5\% BSA in 1M PBS. Sera obtained from the mice after two-week post-immunization, were diluted in PBS, added to the plate and cultured at room temperature for $2 \mathrm{~h}$. After complete washing in PBS, the wells were incubated with 1:3000 dilution of HRP-labeled goat anti-mouse polyclonal antibody (Sigma-Aldrich Corp., MO, USA) at room temperature for $1 \mathrm{~h}$. The plate was totally washed for $30 \mathrm{~min}$, stained with 
Ophenylenediamine at room temperature for $15 \mathrm{~min}$ and terminated with $50 \mu 12 \mathrm{M} \mathrm{H}_{2} \mathrm{SO}_{4}$. The ELISA wells were checked at $490 \mathrm{~nm}$ under a ELISA reader. The concentration of antibody was measured in comparison with standard sample diluents.

In Vivo Tumor Loaded Experiment. To verify the effect of HSP72-P/AFP-P immunization to decrease the growth rate of loaded tumors, BALB/C mice in each group were injected s.c. with $1 \times 10^{6} \mathrm{H} 22$ cells in the left flank on day 0 , and injected s.c. in the right flank with different immunization treatments on day 7, 14, and 21 as above described, while PBS was used as a control. The growth of tumor was checked everyday. The size of tumor was examined with calipers in long and short dimensions every 3 days after inoculation. The volume of tumor was assessed by the formula: $\mathrm{V}=\left(\mathrm{a}^{2} \mathrm{~b}\right) / 2$.

Statistical Anlysis. All data were showed as means \pm S.D. The frequencies of IFN- $\gamma$ producing splenocytes were assessed by $\chi^{2}$ test. The Student's $t$ test was conducted to explain the significant differences between tumor volumes of each animal groups. Statistically significance was considered as $\mathrm{P}<0.05$.

\section{Results}

Cross-linking Complex HSP72-P/AFP-P Induce Specific AFP T-cells Immunity and antiAFP Antibody in Mice

Since T cells play an important function in anti-tumor effect, we detected the frequency of IFN- $\gamma$ producing specific $\mathrm{T}$ cell after HSP72-P/AFP-P immunization. As shown in Table 1, vaccination with HSP72-P/AFP-P cross-linking complexes induced stronger $\mathrm{T}$ cells responses than those with AFP-P or HSP72-P immunization alone $(\mathrm{P}<0.01)$. No significant difference was found between the groups vaccination with AFP-P, HSP72-P and PBS alone $(\mathrm{P}>0.05)$. Our results suggested that HSP72-P/AFP-P immunization produced higher specific AFP T cells reaction, while vaccination with AFP-P or HSP72-P alone failed to elicit a measurable T cell response.

The concentration of anti-AFP antibody in the immunized mice was examined by ELISA. Two weeks after the last immunization, HSP72-P/AFP-P vaccinated mice generated much higher level of specific AFP antibody than those immunized with AFP-P or HSP72-P alone (P < 0.01) (Table 1). No significant difference was seen between the groups vaccination with AFP-P, HSP72-P and PBS alone $(\mathrm{P}>0.05)$. This result suggested that the conjugation of AFP-P with HSP72-P definitely induced specific antibody responses, while immunization with either AFP-P or HSP72-P alone failed to produce a detectable antibody response.

Table 1 IFN- $\gamma$-producing spots of splenocytes and concentration of anti-AFP antibody in mice

\begin{tabular}{|c|c|c|c|c|}
\hline Groups & HSP72-P/AFP-P & AFP-P & HSP72-P & PBS \\
\hline $\begin{array}{c}\text { Spots } \\
\left(10^{6} \text { cells }\right)\end{array}$ & $116.40 \pm 5.70^{\mathrm{a}}$ & $12.42 \pm 6.37^{\mathrm{b}}$ & $11.35 \pm 5.62^{\mathrm{c}}$ & $10.26 \pm 3.45^{\mathrm{d}}$ \\
\hline $\begin{array}{c}\text { Anti-AFP } \\
(\mu \mathrm{g} / \mathrm{mL})\end{array}$ & $143.62 \pm 7.38^{\mathrm{a}}$ & $8.42 \pm 3.65^{\mathrm{b}}$ & $7.83 \pm 3.46^{\mathrm{c}}$ & $6.62 \pm 2.41^{\mathrm{d}}$ \\
\hline \multicolumn{4}{|c|}{${ }^{\mathrm{a}} \mathrm{P}<0.05$, vs group c; ${ }^{\mathrm{b}} \mathrm{P}<0.05$, vs group d; ${ }^{\mathrm{c}} \mathrm{P}>0.05$, vs group d } \\
\hline
\end{tabular}

HSP72-P/AFP-P Immunization Produced Anti-Tumor Effect in Mice Loaded with Tumors

In order to confirm the specific AFP T cells response induced by HSP72-P/AFP-P complex, we examined the therapeutic effect of different vaccines to elicit regression of established AFP expressing $\mathrm{H} 22$ cancer cells in vivo. In the study, BALB/C mice were inoculated s.c. with $1 \times 10^{6}$ H22 cells in the left flank, and immunized s.c. with HSP72-P/AFP-P, AFP-P and HSP72-P in the right flank on days 7 , day 14 and day 21. Tumor growth rate was examined for four weeks since day seven after tumor vaccination (Table 2). The data indicated that HSP72-P/AFP-P vaccination could decrease the tumor size in tumor loaded mice significantly than AFP immunization alone, suggesting that the immunization with HSP72-P/AFP-P elicited a distinct therapeutic reaction on H22 hepatocarcinoma cells loaded in vivo. 
Table 2 Tumor growth in mice loaded with H22 hepatocellular carcinoma cells

\begin{tabular}{|c|c|c|c|}
\hline Groups & $\begin{array}{c}\text { No. tumor- } \\
\text { bearing/No. } \\
\text { mice loaded }\end{array}$ & $\begin{array}{c}\text { 10 days after tumor } \\
\text { loaded /Tumor size } \\
\left(\mathbf{m m}^{\mathbf{3}}\right)\end{array}$ & $\begin{array}{c}\mathbf{2 0} \text { days after tumor } \\
\text { loaded /Tumor size } \\
\left(\mathbf{m m}^{\mathbf{3}}\right)\end{array}$ \\
\hline HSP72-P/AFP-P & $1 / 10$ & $25.46 \pm 6.34^{\mathrm{a}}$ & $29.74 \pm 6.25^{\mathrm{a}}$ \\
\hline AFP-P & $10 / 10$ & $138.63 \pm 8.35^{\mathrm{b}}$ & $632.56 \pm 10.47^{\mathrm{b}}$ \\
\hline HSP72-P & $10 / 10$ & $136.47 \pm 9.63^{\mathrm{c}}$ & $630.84 \pm 11.93^{\mathrm{c}}$ \\
\hline PBS & $10 / 10$ & $137.85 \pm 11.65^{\mathrm{d}}$ & $631.94 \pm 12.36^{\mathrm{d}}$ \\
\hline \multicolumn{3}{|c}{} \\
\hline \multicolumn{3}{|c}{$\mathrm{P}<0.05$, vs group $\mathrm{b} ;{ }^{\mathrm{b}} \mathrm{P}>0.05$, vs group d; ${ }^{\mathrm{c}} \mathrm{P}>0.05$, vs group $\mathrm{d}$}
\end{tabular}

\section{Discussion}

Most concerns have focused on cancer immunity which aims to elicit hosts' immune protection against tumor cells. $80 \%$ of primary liver cancers express a high rate of alpha-fetoprotein (AFP), which may act as a target for cancer therapy [3-5]. AFP is an oncofetal antigen during HCC progression which could induce weak reproducible antitumor effect. Therefore, a vaccine specifically targeting AFP is desirable. AFP oncofetal antigen is essential to the promotion and maintenance of cancer cell transformation, which could be an ideal target antigen for AFP vaccine [6,7]. However, AFP immunization has been restricted by improper antigen-specific immune reaction. Therefore, therapies to generate more potential immunotherapy need to be completely explored.

Heat shock proteins (HSP) have been verified to serve as potential immunoadjuvant to increase antigen-specific tumor reaction [8,9]. Thus, an innovative method combining with HSP families will probably produce more potential antitumor immunity $[10,11]$. In the present study, we studied whether AFP epitope peptide immunogenicity could be enhanced by HSP72 functional peptide and whether HSP72 functional peptide could increase the potency of AFP peptide immunity and assessed the immune reaction elicited by HSP72-P/AFP-P. We constructed a tumor vaccine including HSP72$\mathrm{P}$ and AFP peptide by way of glutaraldehyde cross-linking. Our results showed that AFP peptide and HSP72 functional peptide exhibited distinct enhancement in AFP specific $\mathrm{CD}^{+} \mathrm{T}$ cells response and encouraging antitumor immunity against AFP expressing tumors. Primimg mice with the reconstructed vaccine, we elicited robust strong protective immunity. Therefore, cross-linking HSP72 functional peptide and AFP peptide provides a promise immunotherapy for hepatocellular carcinoma.

Interestingly, the HSP72-P/AFP-P cross-linking peptide vaccine elicited not only the specific cellular immunoprotection, but also a high concentration of anti-AFP antibody, whereas vaccination with AFP-P or HSP72-P alone failed to elicit a measurable humoral response. The above results indicated that AFP-P, by itself, is not immunogenic or weakly immunogenic and conjugating to HSP72 functional peptide renders it highly immunogenic.

One reasonable explanation is that the cross-linking of HSP72 functional peptide with AFP peptide may bring about activation of specific AFP $\mathrm{CD}^{+} \mathrm{T}$ cells in vivo via the "cross-priming" mechanisms $[12,13]$. As a complicated process, cross-priming demands the post-processing of the exogenous antigen by way of various cellular compartments of the antigen presenting cells (APCs). It has been confirmed that HSP complexes could accompany with proteins or peptides and directly anchor peptides onto the MHC I molecule of APCs. Through this procedure, HSP chaperoned peptide complexes are considered to induce tumor-specific T cell immunity [14]. It is presumed that HSP72-P/AFP-P can react with different receptors on the membrane of APCs giving rise to crosspriming antigen. The different receptors are likely to account for the increasing specific AFP CD8 ${ }^{+}$ T cell reaction in mice immunized with HSP72-P/AFP-P.

In the study, we generated a strategy to construct an innovative protein vaccine. Cross-linking of HSP72 functional peptide and AFP peptide with lower concentration of glutaraldehyde not only established the HSP72-P/AFP-P complex but also preserve the activity of HSP72 immunoadjuvant and immunogenity of AFP antigen, which was confirmed by the strong ex vivo and in vivo antitumor effect. In the present study, tumor loaded experiment indicated that cross-linking protein 
vaccine HSP72-P/AFP-P induced stronger specific antitumor effect on AFP-producing H22 hepatocarcinoma cells than AFP-P immunization. Results suggested that the immunogenicity of AFP peptide could be greatly enhanced by HSP72 functional peptide and immunization with HSP72 functional peptide could elicit humoral and T cells response to AFP peptide.

\section{Summary}

In conclusion, our results suggest that HSP72 functional peptide and AFP peptide cross-linking vaccine could produce an distinct antitumor protection on AFP-expressing hepatocarcinoma cells via eliciting specific $\mathrm{AFP} \mathrm{CD}^{+} \mathrm{T}$ cell responses. Therefore, the cross-linking vaccine by combining AFP peptide and HSP72 functional peptide is a encouraging therapy for AFP positive hepatocellular carcinoma.

\section{Acknowledgments}

This work is supported by Scientific Research Program Funded by Shaanxi University of Chinese Medicine and Shaanxi Provincial Education Department (No.2007JK233, 2009xj02, 2010JK484, 14JS025), the Scientific Research Program of Shaanxi Administration of Traditional Chinese Medicine (No.15-SCJH001) and the Natural Science Basic Research Plan in Shaanxi Province of China (No.2016JM8023, 2016JM8150).

\section{References}

[1] J.H. Zhong, H. Li, L.Q. Li, X.M. You, Y. Zhang, Y.N. Zhao, et al. Adjuvant therapy options following curative treatment of hepatocellular carcinoma: A systematic review of randomized trials, Eur. J. Surg. Oncol. 38 (2012) 286-295.

[2] J.A. Marrero, and K.S. Henley, The role of serum biomarkers in hepatocellular carcinoma surveillance, Gastroenterol. Hepatol. (N Y). 7 (2011) 821-823.

[3] L.H. Butterfield, A. Ribas, V.B. Dissette, Y. Lee, J.Q. Yang, P. De la Rocha, et al. A phase I/II trial testing immunization of hepatocellular carcinoma patients with dendritic cells pulsed with four alpha-fetoprotein peptides, Clin. Cancer Res. 12 (2006) 2817-2825.

[4] Y. Liu, L.H. Butterfield, X. Fu, Z. Song, X. Zhang, C. Lu, et al. Lentivirally engineered dendritic cells activate AFP-specific $\mathrm{T}$ cells which inhibit hepatocellular carcinoma growth in vitro and in vivo, Int. J. Oncol. 39 (2011) 245-253.

[5] J. Cany, B. Barteau, L. Tran, V. Gauttier, I. Archambeaud, J.P. Couty, et al. AFP-specific immunotherapy impairs growth of autochthonous hepatocellular carcinoma in mice, J. Hepatol. 54 (2011) 115-121.

[6] M.M. Rodríguez, S.M. Ryu, C. Qian, M. Geissler, C. Grimm, J. Prieto, et al. Immunotherapy of murine hepatocellular carcinoma by alpha-fetoprotein DNA vaccination combined with adenovirus-mediated chemokine and cytokine expression, Hum. Gene Ther. 19 (2008) 753-759.

[7] L.H. Butterfield, A. Ribas, D.M. Potter, and J.S. Economou, Spontaneous and vaccine induced AFP-specific T cell phenotypes in subjects with AFP-positive hepatocellular cancer, Cancer Immunol. Immunother. 56 (2007) 1931-1943.

[8] X.P. Wang, H.P. Lin, Q.X. Wang, Y. Gu, Specific antitumor immunity induced by cross-link complex heat shock protein 72 and alpha-fetoprotein, Cancer Biotherapy \& Radiopharmaceuticals. 27 (2012) 189-197.

[9] X.P. Wang, H.P. Lin, Q.X. Wang, S.Z. Li, L.J. Sun, Y.X. Yang, HSP72 and gp96 in gastroenterological cancers, Clinica Chimica Acta. 417 (2013) 73-79. 
[10] X.P. Wang, Q.X. Wang, H.P. Lin, Y.L. Wang, Y. Yang, Glycoprotein 96 and $\alpha$-fetoprotein cross-linking complexes elicited specific antitumor immunity, Cancer Biotherapy \& Radiopharmaceuticals. 28 (2013) 406-414.

[11]Z. Li, X.P. Wang, H.P. Lin, B. Xu, Q. Zhao, B.N. Qi, et al. Anti-tumor immunity elicited by cross-linking vaccine heat shock protein 72 and alpha-fetoprotein epitope peptide, Neoplasma. 62 (2015) 713-721.

[12] M. Santone, S. Aprea, T.Y. Wu, M.P. Cooke, M.L. Mbow, N.M. Valiante, et al. A new TLR2 agonist promotes cross-presentation by mouse and human antigen presenting cells, Hum. Vaccine Immunother. 11 (2015) 2038-2050.

[13] Y. Zhang, G. Chen, Z. Liu, S. Tian, J. Zhang, C.D. Carey, et al. Genetic vaccines to potentiate the effective $\mathrm{CD} 103^{+}$dendritic cell-mediated cross-priming of antitumor immunity, J Immunol. 194 (2015) 5937-5947.

[14] Y.J. Zhou, M.N. Messmer, R.J. Binder, Establishment of tumor-associated immunity requires interaction of heat shock proteins with CD91, Cancer Immunol. Res. 2 (2014) 217-228. 